\title{
Upregulation of IL-11, an IL-6 Family Cytokine, Promotes Tumor Progression and Correlates with Poor Prognosis in Non-Small Cell Lung Cancer
}

\author{
Meng Zhao ${ }^{a}$ Yahui Liu ${ }^{\mathrm{a}}$ Ran Liub Jin Qic Yongwang Hou \\ Jiao Chang ${ }^{\mathrm{a}} \quad$ Li Ren $^{\mathrm{a}}$
}

\begin{abstract}
aDepartment of Clinical Laboratory, Tianjin Medical University Cancer Institute and Hospital, National Clinical Research Center for Cancer, Key Laboratory of Cancer Prevention and Therapy, Tianjin's Clinical Research Center for Cancer, National Human Genetic Resources Sharing Service Platform, Tianjin, bepartment of Immunology, Tianjin Key Laboratory of Cellular and Molecular Immunology, Key Laboratory of Educational Ministry of China, School of Basic Medical Sciences, Tianjin Medical University, Tianjin, 'Radiology Department, Tianjin Medical University Cancer Institute and Hospital, National Clinical Research Center for Cancer, Key Laboratory of Cancer Prevention and Therapy, Tianjin's Clinical Research Center for Cancer, National Human Genetic Resources Sharing Service Platform, Tianjin, P.R. China
\end{abstract}

\section{Key Words}

IL-11 • Hypoxia $・$ EMT $・$ NSCLC

\begin{abstract}
Background/Aims: Cytokines are key players in tumorigenesis and are potential targets in cancer treatment. Although IL-6 has attracted considerable attention, interleukin 11 (IL-11), another member of the IL- 6 family, has long been overlooked, and little is known regarding its specific function in non-small cell lung cancer (NSCLC). In this study, we explored IL-11's role in NSCLC and the detailed mechanism behind it. Methods: Cell proliferation in response to IL11 was determined by colony formation, BrdU incorporation and MTS (3-(4,5-dimethylthiazol2-yl)-5-(3-carboxymethoxyphenyl)-2-(4-sulfophenyl)-2H-tetrazolium) assay. Cell motility was measured by Transwell and wound healing assays. NSCLC xenograft models were used to confirm oncogenic function of IL-11 in vivo. Immunohistochemical staining and western blot assay were performed to detect epithelial-mesenchymal transition (EMT) markers and cell signaling pathway alterations. Eighteen NSCLC patients and 5 normal lung samples were collected together with data from an online database to determine the link between IL-11 expression and malignant progression. Results: We observed that IL-11 was upregulated in NSCLC samples compared with normal tissue samples and correlated with poor prognosis. Data from in vitro and in vivo models indicated that IL-11 promotes cell proliferation and tumorigenesis. Cell migration and invasion were also enhanced by IL-11. Epithelialmesenchymal transition (EMT) was also observed after IL-11 incubation. Furthermore, IL-11
\end{abstract} M. Zhao and Y. Liu contributed equally to this work. 
activated AKT and STAT3 in our experimental models. In addition, we observed that hypoxia induced IL-11 expression in NSCLC cells. Deferoxamine (DFX) or dimethyloxalylglycine (DMOG) induced hypoxia-inducible factor 1-alpha (HIF1 $\alpha$ ) upregulation, which enhanced IL11 expression in NSCLC cells. Conclusions: Taken together, our results indicate that IL-11 is an oncogene in NSCLC, and elucidating the mechanism behind it may provide insights for NSCLC treatment.

(C) 2018 The Author(s)

Published by S. Karger AG, Basel

\section{Introduction}

Tumorigenesis is the interplay between "seed and soil." Although cell-autonomous defects, such as aberrant regulation of oncogenes and tumor suppressors, promote proliferation, resistance to apoptosis and metastasis of cancer, the tumor microenvironment is increasingly recognized as a crucial player in tumor formation [1]. Lung cancer is the leading cause of cancer related deaths worldwide. In the United States, it is estimated that lung cancer accounts for 222, 500 new cases and 155, 870 deaths in 2017 [2]. Due to vulnerability to numerous pathogens, oxidants and toxicants, the human lung suffers chronic injury and inflammation, which is a well-known risk factor for lung cancer [3].

The cytokine network is the bridge connecting the inflammatory environment to cancer cells. Clinical studies indicated that increased concentration of cytokines, such as the IL-6 family of cytokines, are strongly associated with poor prognosis and may be potential targets in cancer therapy [4].

The interleukin 6 family of cytokines comprises of IL-6, IL-11, OSM, CT-1, CLC, LIF and others that have similar helical cytokine structure and receptor subunit makeup. IL-6 family cytokines share a receptor subunit, gp130. By formation of a larger complex, the JAK-STAT3 pathway, RAS-ERK and PI3K-AKT pathways can be activated by gp130. Although IL-6 has attracted the most attention, a recent study indicated the importance of IL-11 in tumorigenesis [5]. In this study, we systematically evaluated the IL-11 role in NSCLC to provide a deep understanding of the mechanism of the cytokine.

\section{Materials and Methods}

Cell culture, lentivirus, plasmids and reagents

Human NSCLC cell lines, A549 and H1299, were obtained from the Type Culture Collection of Chinese Academy of Sciences and maintained in culture according to their recommendations. The $\mathrm{O}_{2}$ concentration was maintained at $0.5 \%$ throughout the course of the hypoxic experiments.

IL-11 expression lentivirus and plasmid were obtained from Shanghai Genechem Co., Ltd. IL-11shRNA and scramble lentivirus were obtained from Shanghai Genepharma Co., Ltd. IL-11-shRNA1 and IL-11-shRNA2 target sequences were TGCACAGCTGAGGGACAAATT and TGAGCCTGTGGCCAGATACAG, respectively. Virus supernatant was incubated with target cells for 12 hours with $8 \mu \mathrm{g} / \mathrm{ml}$ polybrene following the manufacturer's instructions. Infected cells were selected in puromycin, as optimized for each cell line. DFX and DMOG were purchased from Sigma-Aldrich. IL-11 antibody for neutralization was obtained from R\&D systems (MAB218).

\section{IL-11 concentration}

IL-11 concentration in cell culture supernatant was determined by the Human IL-11 Immunoassay ELISA kit (R\&D Systems) following the manufacturer's instructions. Briefly, $100 \mu \mathrm{l}$ of assay diluent and 100 $\mu \mathrm{l}$ of standard or sample per well were added to the microplate and incubated for 2 hours. After being washed 4 times, the microplate was later incubated with $200 \mu \mathrm{l}$ of IL-11 conjugate for 3 hours. After 4 washes, $200 \mu \mathrm{l}$ of substrate solution was added and incubated for 30 minutes. Absorbance was determined at $450 \mathrm{~nm}$. 


\section{Cellular Physiology Cell Physiol Biochem 2018;45:2213-2224 \begin{tabular}{ll|l} 
and Biochemistry Published onlıne: March 15, 2018 & $\begin{array}{l}\text { (c) } 2018 \text { The Author(s). Published by S. Karger AG, Basel } \\
\text { www.karger.com/cpb }\end{array}$ \\
\hline
\end{tabular} \\ Zhao et al.: IL-11 Promotes NSCLC Progression}

\section{Colony formation assay}

Five hundred of A549 or H1299 cells were plated in triplicate in 6-well dishes using the appropriate growth media for each cell line. Media was replaced every two days. Colonies were counted after 7-10 days.

\section{Cell proliferation assay}

For the BrdU incorporation assay, the cell proliferation assay was performed as described previously [6]. Briefly, cells infected with shIL11 or scramble control were seeded in triplicate in 96-well plates and incubated with complete medium for 24 h. For IL-11 incubation, A549 and H1299 cells were incubated with IL-11 with the indicated concentrations for 24 hours. Next, cell proliferation was determined by a Cell Proliferation ELISA, BrdU kit (Roche Applied Science, USA), as per the manufacturer's instructions. Each point represents a mean value and standard of three experiments with three replicates.

For the MTS assay, pretreated NSCLC cells (5, 000 cells / well) were seeded in 96-well plates. Twelve hours later, $10 \mu \mathrm{l}$ of $0.5 \mathrm{mg} / \mathrm{ml}$ MTS reagent (Promega) was added to each well. The cells were incubated at $37^{\circ} \mathrm{C}$ for 2 hours. Next, the absorbance was detected at $570 \mathrm{~nm}$ on a $\mu Q$ uant Universal Microplate Spectrophotometer (Bio-Tek Instruments, Winooski, USA). The absorbance was recorded as Day 0. Next, absorbance at Days 2, 3, 4 and 5 were recorded, respectively. The absorbance was normalized to Day 0 data, and the proliferation curve was drawn.

\section{In vitro invasion and migration assay}

The invasion and migration assays were described previously [7]. For the Transwell migration assay, NSCLC cells were trypsinized and placed in the upper chamber of each insert (Corning, Cambridge, USA) containing the non-coated membrane. For the invasion assay, cells were placed on the upper chamber of each insert coated with $40 \mu \mathrm{l}$ of Matrigel (BD biosciences), which was diluted to $4 \mu \mathrm{g} / \mu \mathrm{l}$ with serumfree medium. Next, medium supplemented with $20 \%$ fetal bovine serum $(600 \mu \mathrm{l})$ was added to the lower chambers. After 24 hours of incubation at $37^{\circ} \mathrm{C}$, the upper surface of the membrane was wiped with a cotton tip, and the cells attached to the lower surface were stained for $10 \mathrm{~min}$ with crystal violet. Cells in five random fields of view at $100 \times$ magnification were counted and expressed as the average number of cells per field of view. All assays were performed in triplicate.

Wound healing assay

NSCLC cells were 95\%-100\% confluent for the wound healing assay. Wounds were generated with a pipette tip, and images of wound healing (migration) were taken at the indicated time intervals.

\section{Immunofluorescence analysis}

The immunofluorescence assay was described previously [7]. Briefly, cells were grown on glass coverslips, fixed with 4\% paraformaldehyde for $15 \mathrm{~min}$, and blocked with phosphate-buffered saline (PBS) containing 5\% fetal bovine serum for $60 \mathrm{~min}$. Cells were incubated with the primary antibody in PBS at $4^{\circ} \mathrm{C}$ overnight, washed three times with PBS, incubated with Alexa Fluor 488 or Alexa Fluor 546-labeled secondary antibody (Invitrogen) in PBS for $1 \mathrm{~h}$ and analyzed using a confocal fluorescence microscope (FV1000-D, Olympus, Tokyo, Japan).

\section{RNA isolation and qRT-PCR assay}

RNA isolation and qRT-PCR assay were described previously [7]. Briefly, total RNA was isolated using Trizol reagent (Invitrogen, Carlsbad, USA). Total RNA $(2 \mu \mathrm{g})$ was used for the synthesis of first-strand cDNA using M-MLV reverse transcriptase (Invitrogen, Beijing, China). Quantitative real-time PCR was performed using the SYBR green mix (Applied Biosystems). The reactions were performed with a 7500 Fast Real-Time PCR System (Applied Biosystems). The data were displayed as $2^{-\Delta \Delta C t}$ values and were representative of at least three independent experiments. Sequences of the RT-PCR primers were as follows (5'- 3'): GAPDH, GGAGCGAGATCCCTCCAAAAT and GGCTGTTGTCATACTTCTCATGG;

IL-11, CGAGCGGACCTACTGTCCTA and GCCCAGTCAAGTGTCAGGTG; E-cadherin, CGAGAGCTACACGTTCACGG and GGGTGTCGAGGGAAAAATAGG; vimentin, GACGCCATCAACACCGAGTT and CTTTGTCGTTGGTTAGCTGGT; N-cadherin, TCAGGCGTCTGTAGAGGCTT and ATGCACATCCTTCGATAAGACTG; ZO-1, CAACATACAGTGACGCTTCACA and CACTATTGACGTTTCCCCACTC; claudin-1, CCTCCTGGGAGTGATAGCAAT and GGCAACTAAAATAGCCAGACCT; 


\section{Cellular Physiology Cell Physiol Biochem 2018;45:2213-2224 \\ and Biochemistry Published ontune: Varch 15, 2018 \begin{tabular}{l|l} 
DOI: 10.1159/000488166 2018 The Author(s). Published by S. Karger AG, Basel \\
www.karger.com/cpb
\end{tabular} \\ Zhao et al.: IL-11 Promotes NSCLC Progression}

snail, TCGGAAGCCTAACTACAGCGA and AGATGAGCATTGGCAGCGAG; slug, CGAACTGGACACACATACAGTG and CTGAGGATCTCTGGTTGTGGT.

\section{NSCLC xenograft models}

Female athymic nude mice were purchased from the Academy of Military Medical Science (Beijing, China). All mouse studies were approved by the Animal Ethics Committee of Tianjin Medical University. All animals were 4-6 weeks of age at the time of injection. A549-scramble or A549-shIL-11-1 cells with luc2 reporter were trypsinized, washed, resuspended in Hanks' balanced salt solution (HBSS; Gibco) and injected into the right flanks subcutaneously $\left(5 \times 10^{6}\right.$ cells/animal $)$ or the tail veins $\left(2 \times 10^{6}\right.$ cells/animal $)$ in mice.

\section{Protein extraction and western blot analysis}

Whole-cell protein was isolated as described previously [7]. Briefly, for tissue protein extraction, the tumor tissues were homogenized in ice-cold RIPA (radioimmunoprecipitation assay) lysis buffer (Millipore). The tissue lysates were incubated at $4^{\circ} \mathrm{C}$ for 1 hour with rotation followed by clarification of tissue debris by centrifugation at 12, $000 \mathrm{rpm}$ for 10 minutes. The protein concentration of tumor extracts was determined using the BCA Protein Assay Kit (Pierce). Western blotting was performed as previously described [7]. Briefly, The following antibodies to E-cadherin (Cell Signaling), vimentin (Cell Signaling), snail (Cell Signaling), slug (Cell Signaling), ZO-1 (Cell Signaling), N-cadherin (Cell Signaling), claudin-1 (Cell Signaling), $\beta$-actin (Sigma Aldrich), total STAT3 (Abcam), pSTAT3 (Ser727) (Abcam), pSTAT3 (Tyr705) (Abcam), pSTAT (Tyr694) (Cell Signaling), total AKT (Cell Signaling), pAKT (Ser473) (Cell Signaling), pAKT (Thr308) (Cell Signaling), and pERK (Cell Signaling) were applied for protein detection. Antibody binding was revealed using an HRPconjugated anti-rabbit IgG or anti-mouse IgG secondary antibody (Sigma). Antibody complexes were detected using Immobilon Western Chemiluminescent HRP Substrate (Millopore) and exposure to Tanon 6200 Luminescent Imaging Workstation (Tanon Science \& Technology Co., Ltd., Shanghai, China).

\section{Statistical analysis}

Data are reported as mean values \pm SD. Biochemical experiments were performed in triplicate, and a minimum of three independent experiments were evaluated. Differences were assessed for statistical significance by an unpaired two-tailed $t$ test by the log rank test (for Kaplan-Meier plots). The p values were considered as follows: ${ }^{*} \mathrm{p}<0.05 ;{ }^{* *} \mathrm{p}<0.01$.

\section{Results}

\section{IL-11 promotes NSCLC cell proliferation in vitro and tumorigenesis in vivo}

Previous studies indicated that IL-11 participates in cell proliferation regulation, but the specific role of IL-11 in NSCLC has not been thoroughly elucidated to date. To investigate IL-11's role in NSCLC, we first established stable IL-11 knockdown NSCLC cell lines. A549 and $\mathrm{H} 1299$ cells were infected with lentivirus expressing IL-11 shRNA. Two different target sequences were applied to avoid off-target effects. The knockdown efficiency was confirmed by qRT-PCR and ELISA assay (Fig. $1 \mathrm{~A}$ and B). Then, we examined whether IL-11 loss affected A549 and H1299 cell proliferation. Colony formation assay was performed, and as we expected, IL-11-inhibited cells produced less and smaller colonies compared with control cells (Fig. 1C and 1D). To further confirm these results, we determined the cell proliferation curve of A549 and H1299 cells. As is shown in Fig. 1E, A549-shIL11 cells exhibited suppressed proliferation compared with scramble control cells. Similar results were obtained from H1299-shIL11 cells (Fig. 1F). The thymidine analog, BrdU (5-Bromo-2'-Deoxyuridine), can naturally incorporate into DNA of proliferating cells during division. We examined whether IL-11 knockdown could affect BrdU incorporation. As is shown in Fig. 1G, inhibition of IL-11 significantly suppressed BrdU incorporation in A549 and H1299 cells.

To further confirm that IL-11 promotes NSCLC cell proliferation, we stably overexpressed IL-11 in A549 and H1299 cells by infecting cells with IL-11 expressing lentivirus. RT-qPCR and ELISA assay confirmed IL-11 overexpression (Fig. 1H and 1I). Consistent with the above 
Fig. 1. IL-11 promotes NSCLC cell proliferation in vitro and tumorigenesis in vivo. (A) RT-qPCR analysis of IL-11 mRNA levels in A549 and H1299 cells infected with shIL111, shIL11-2 or scrambleshRNA. The data are shown as the means $\pm \operatorname{SD}(n=3)$. (B) ELISA analysis of IL-11 concentration in supernatant of A549 and H1299 cells infected with shIL111, shIL11-2 or scrambleshRNA. The data are shown as the means $\pm \operatorname{SD}(n=3)$. (C) Representative graphs of A549 in the colony formation assay. (D) Colony formation of A549 and H1299 cells expressing scramble or shIL11-1 or shIL11-2 shRNA. Colony numbers were counted at day 10 . The data are shown as the means $\pm \operatorname{SD}(n=3)$. (E and F) Proliferation curve of A549 and H1299 cells infected with shIL11-
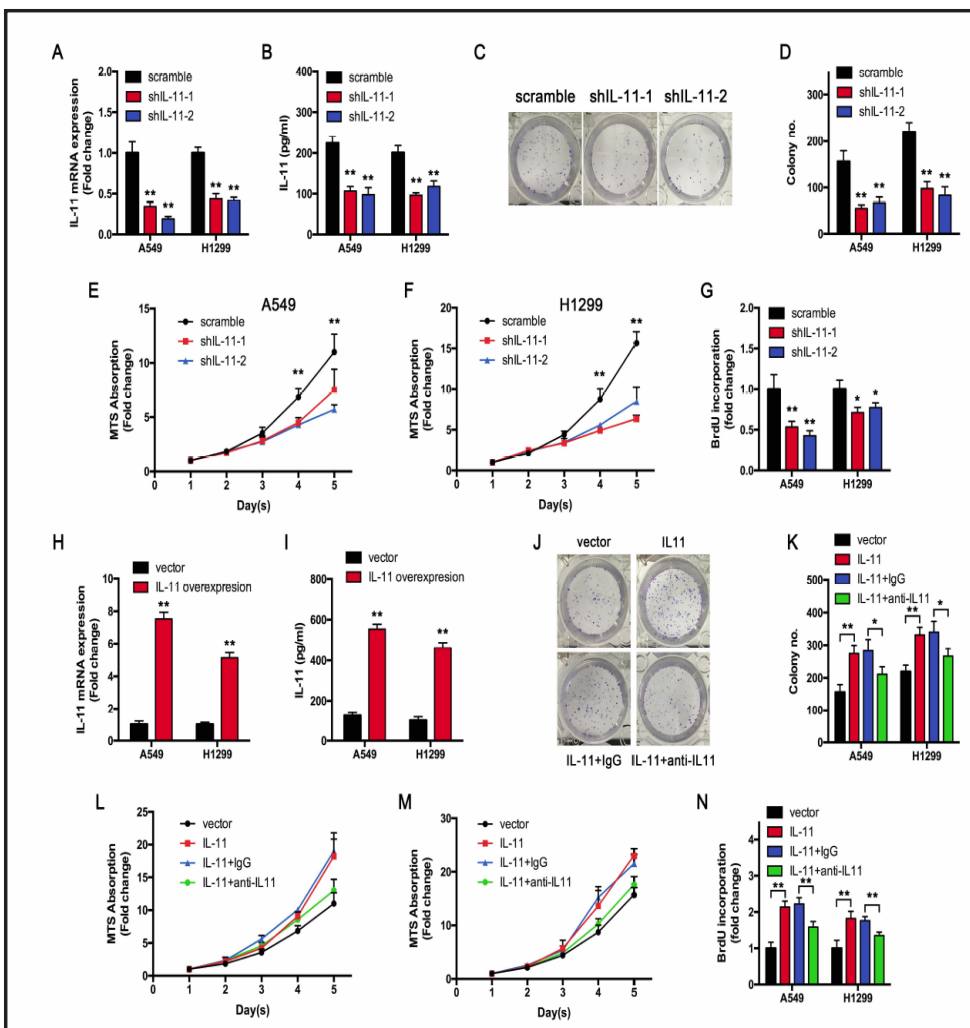

M

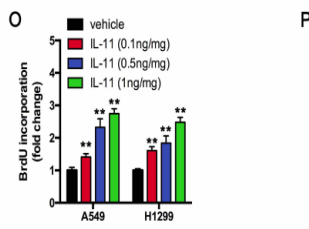

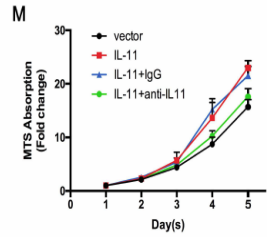

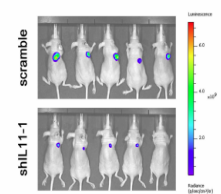

N

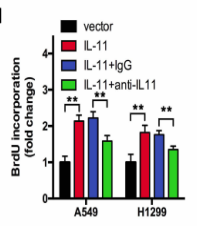

Q

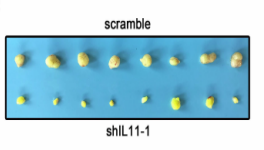
1, shIL11-2 or scrambleshRNA determined by MTS assay. The data are shown as the means \pm SD $(n=3)$. (G) BrdU incorporation assay of A549 and H1299 cells infected with shIL11-1, shIL11-2 or scramble-shRNA. The data are shown as the means \pm SD $(n=3)$. $(H)$ RT-qPCR analysis of IL-11 mRNA levels in A549 and H1299 cells infected with IL-11 expressing or vector lentivirus. The data are shown as the means \pm SD $(n=3)$. (I) ELISA analysis of IL-11 concentration in supernatants of A549 and H1299 cells infected with IL-11 expressing or vector lentivirus. The data are shown as the means \pm SD $(n=3)$. (J and K) Colony formation of A549 and H1299 cells infected with IL-11 or vector lentivirus. The data are shown as the means \pm SD $(n=3)$. (J) Representative graphs of A549 in the colony formation assay. (L and M) Proliferation curve of A549 and H1299 cells infected with IL-11 expressing or vector lentivirus determined by MTS assay. The data are shown as the means \pm SD $(n=3)$. (N) BrdU incorporation assay of A549 and H1299 cells infected with IL-11 expressing or vector lentivirus. The data are shown as the means \pm SD ( $n=3$ ). (0) A549 or H1299 cells incubated with the indicated concentrations of IL-11 for 24 hours. Then, cells were subjected to the BrdU incorporation assay. The data are shown as the means \pm SD $(\mathrm{n}=3)$. (P and Q) Female nude mice were injected with A549-scramble or A549-shIL-11-1 cells with luc2 reporter into the right flanks subcutaneously of mice $\left(5 \times 10^{6}\right.$ cells/animal $)$. Tumor burden was quantified using bioluminescence imaging. Three weeks after injection, the mice were euthanized. $*, \mathrm{P}<0.05, * *, \mathrm{P}<0.01$.

data, both A549-IL11 and H1299-IL-11 cells produced more and larger colonies (Fig. 1J and $1 \mathrm{~K}$ ). Proliferation rates of the two cell lines were also upregulated (Fig. 1L and 1M). Furthermore, IL-11 overexpression enhanced BrdU incorporation in A549 and H1299 cells (Fig. 1N). However, when treated with IL-11 neutralizing antibody, IL-11-induced enhanced proliferation was significantly reduced (Fig. 1J-1N), which suggests that IL-11 acts in an 
autocrine fashion. In addition, incubation of A549 and H1299 cells with IL-11 also enhanced BrdU incorporation in a dose-dependent manner (Fig. 10).

To further verify the oncogenic role of IL-11, we established a subcutaneous xenograft mouse model. A549 cells treated with shIL11-1 or scramble shRNA were injected into the right flanks of female athymic nude mice. As shown in Fig. 1, IL-11 suppression resulted in a decrease of tumor volume and weight (Fig. 1P and 1Q). Taken together, these results confirm the oncogenic role of IL-11 in NSCLC.

\section{IL-11 promotes NSCLC cell invasion and migration in vitro and lung colony formation in} vivo

Activating invasion and metastasis is one of the hallmarks of cancer $[8,9]$. To further investigate the significance of IL-11 in NSCLC, we subsequently explored the role of IL-11 in invasion and metastasis. A549-shIL11-1 and -2 cells were subjected to the Transwell migration assay, which reflects the capacity of cell migration. The results showed that IL-11 knockdown impaired A549 cell migration (Fig. 2A and 2B). Similar results were obtained from H1299 cells (Fig. 2A and 2B). Next, we confirmed the results using a wound healing assay. IL-11 suppression significantly attenuated the migration of A549 cells (Fig. 2D). Matrigel coated boyden chambers were used to assess cell invasiveness. As is shown in

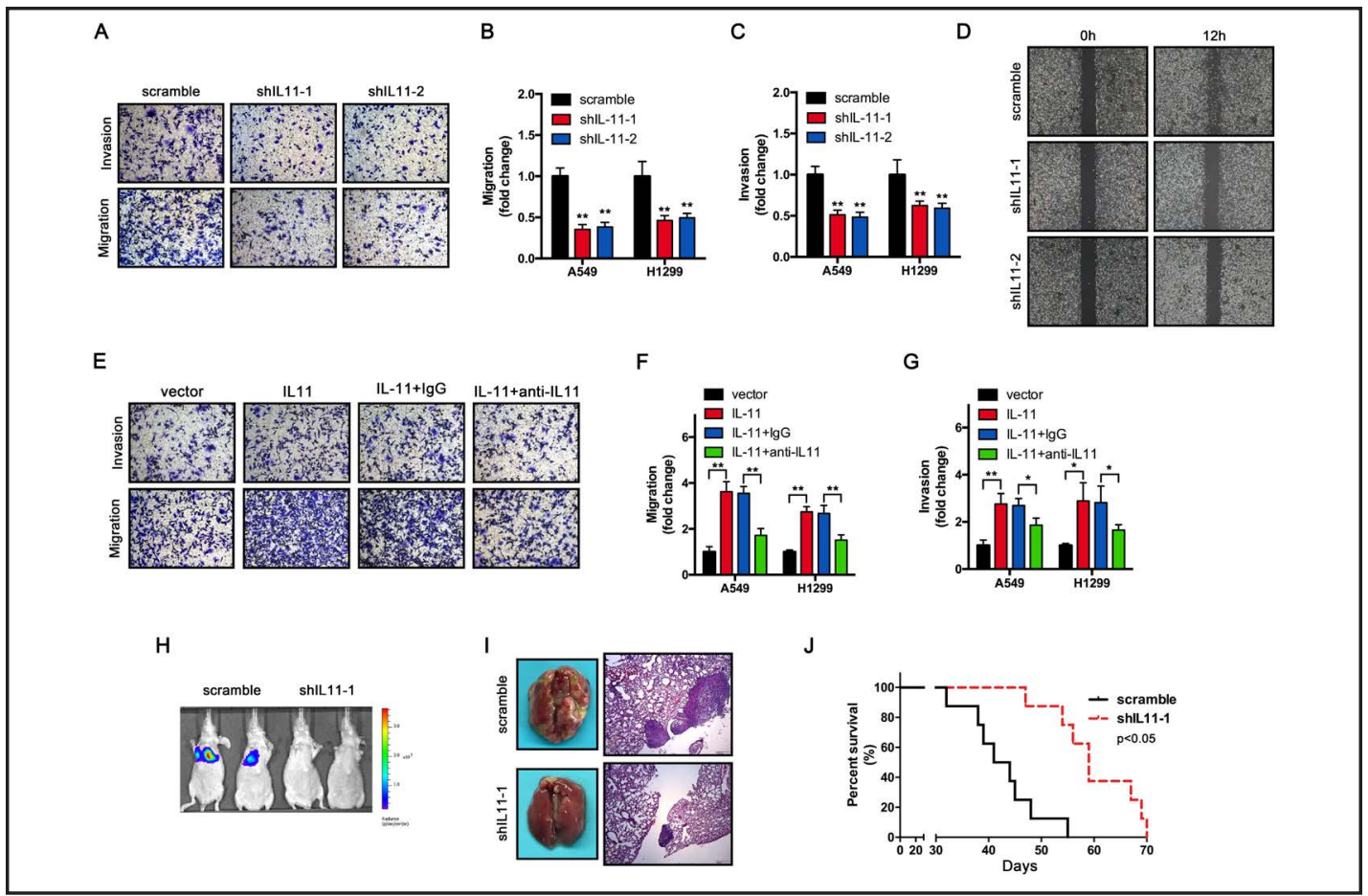

Fig. 2. IL-11 promotes NSCLC cell invasion and migration in vitro and lung colony formation in vivo. (A, B and C) Assays for cell migration or invasion of A549 and H1299 cells infected with shIL11-1, shIL11-2 or scramble-shRNA. All the data were normalized to the results of cells transfected with scramble-shRNA. The data are shown as the means \pm SD ( $n=3)$. (A) Representative graphs of A549 cells in migration or invasion assays. (D) Wound healing assay for A549 and H1299 cells infected with shIL11-1, shIL11-2 or scramble-shRNA. (E, F and G) Assays for cell migration or invasion on A549 and H1299 cells infected with infected with IL-11 expressing or vector lentivirus. All the data were normalized to the results of cells transfected with vector lentivirus. The data are shown as the means \pm SD $(n=3)$. (E) Representative graphs of A549 cells in migration or invasion assays. (H and I) Female nude mice were injected with A549-scramble or A549-shIL-11-1 cells with luc2 reporter into the tail vein of mice $\left(2 \times 10^{6}\right.$ cells/animal). Tumor burden was quantified using bioluminescence imaging. Three weeks after injection, the mice were euthanized. (J) Kaplan-Meier survival curves of mice. *, $\mathrm{P}<0.05, * *, \mathrm{P}<0.01$. 
A

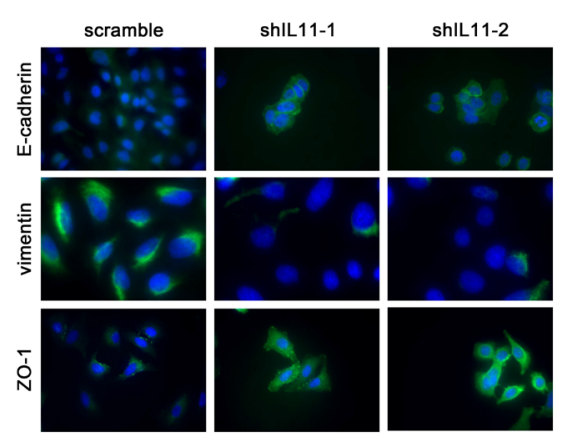

B

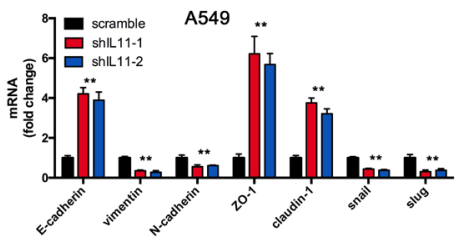

C

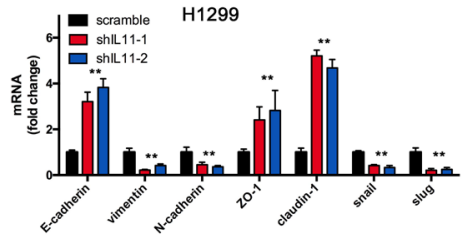

D

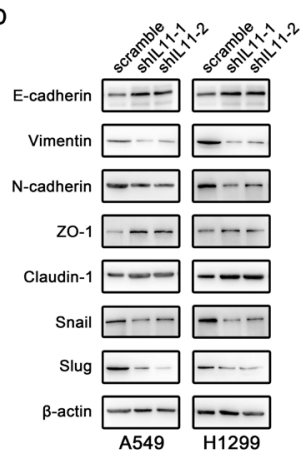

E

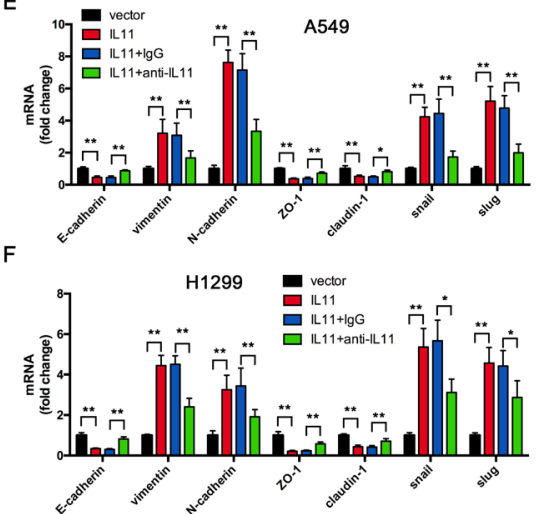

G

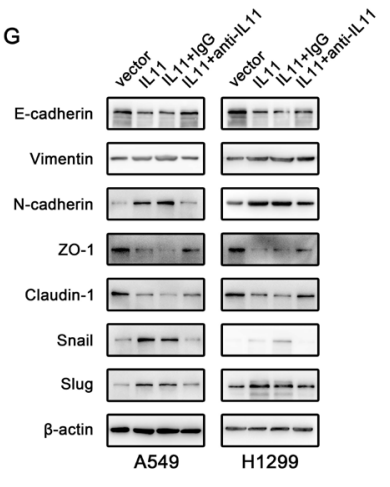

Fig. 3. IL-11 promotes EMT in NSCLC cells. (A) Immunofluorescence analysis of EMT markers in A549 cells infected with shIL11-1, shIL11-2 or scramble-shRNA. (B and C) RT-qPCR analysis of EMT markers in A549 and H1299 cells infected with shIL11-1, shIL11-2 or scramble-shRNA. The data are shown as the means \pm SD. (D) Western blot analysis of EMT markers in A549 and H1299 cells infected with shIL11-1, shIL11-2 or scramble-shRNA. (E and F) RT-qPCR analysis of EMT markers in A549 and H1299 cells infected with IL-11 expressing or vector lentivirus. The data are shown as the means \pm SD. (G) Western blot analysis of EMT markers in A549 and H1299 cells infected with IL-11 expressing or vector lentivirus. * $\mathrm{P}<0.05, * *, \mathrm{P}<0.01$.

Fig. 2A and 2C, both A549 and H1299 cell lines exhibited decreased invasion after IL-11 inhibition.

Overexpression of IL-11 in A549 and H1299 enhanced cell migration in Transwell migration assays (Fig. 2E and 2F). Furthermore, aggravated invasion was also observed in IL11 overexpressed cells (Fig. 2E and 2G). When treated with the IL-11 neutralizing antibody, IL-11-induced enhanced invasion and migration were reversed (Fig. 2E-2G).

To further confirm that IL-11 promotes cell locomotion in vivo, we injected A549shIL11-1 or A549-scramble cells into the tail veins of nude mice. As we expected, A549shIL11-1 cells produced a decreased tumor bearing burden in the lung (Fig. 2H and 2I). Furthermore, inhibition of IL-11 prolonged the survival of tumor bearing mice (Fig. 2J). Taken together, these data indicate that IL-11 may be a potential metastatic promoter in NSCLC.

\section{IL-11 promotes EMT in NSCLC cells}

The epithelial-mesenchymal transition (EMT) is a process by which epithelial cells lose their cell polarity and cell-cell adhesion and gain migratory and invasive properties to become mesenchymal cells [10]. Because the above results indicated that IL-11 positively controls NSCLC cell proliferation and locomotion, we then turned to investigate whether

\section{KARGER}


IL-11 induces EMT to achieve these effects. Ecadherin, claudin-1 and ZO-1 are epithelial markers, whereas vimentin and $\mathrm{N}$-cadherin are mesenchymal markers [11, 12]. We first examined whether IL-11 knockdown could regulate these markers expressed in NSCLC cells.

Immunofluorescence analysis showed that A549 cells infected with shIL11-1 or shIL11-2 expressed more E-cadherin on the cell surface compared with control cells (Fig. 3A). In addition, IL-11 knockdown also upregulated Z0-1 expression (Fig. 3A). In contrast, downregulation of vimentin was observed after IL11 inhibition (Fig. 3A).

Inhibiting IL-11 in A549 or H1299 cells promoted mRNA expression of E-cadherin, claudin-1 and ZO-1, while suppressing mRNA expression of vimentin and $\mathrm{N}$-cadherin (Fig. 3B and 3C). Consistent with mRNA expression, western blot assay showed that IL-11 knockdown augmented E-cadherin, claudin-1 and ZO-1 expression and attenuated vimentin and $\mathrm{N}$-cadherin expression in A549 and H1299 cells (Fig. 3D). Snail and slug are two transcriptional repressors involved in E-cadherin and other epithelial markers regulation [13]. We determined whether IL11 also controlled these transcriptional repressors. Both RT-qPCR and western blot assay dem-

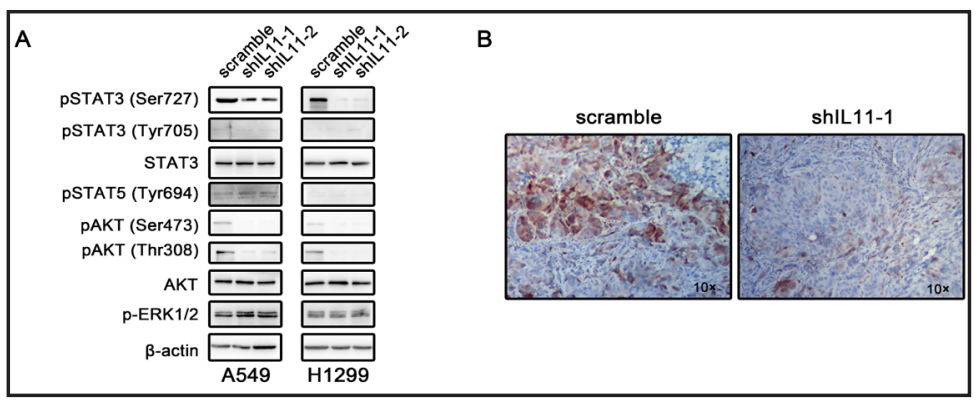

Fig. 4. IL-11 activates STAT3 and AKT pathway in NSCLC cells. (A) Phosphorylation of STAT3, STAT5, AKT and ERK1/2 in A549 and H1299 cells infected with shIL11-1, shIL11-2 or scramble-shRNA was analyzed by western blot. (B) Xenograft tumor sections (A549) were also examined for levels of p-STAT3 by IHC.

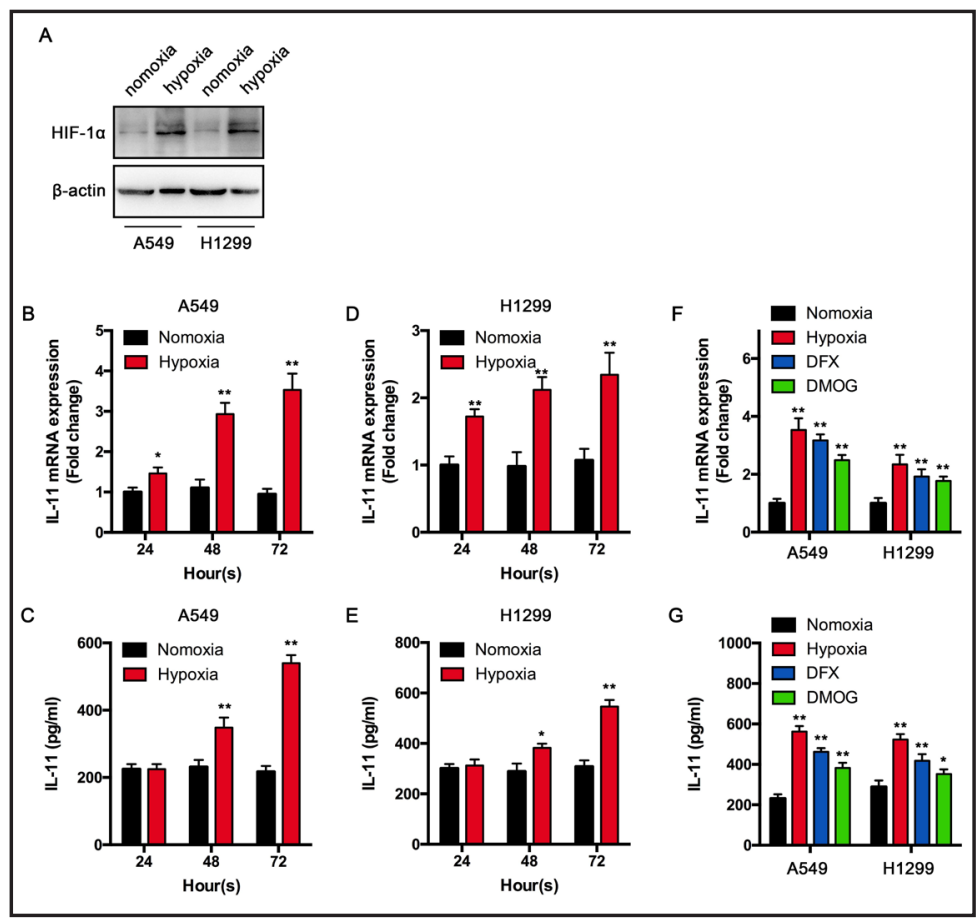

Fig. 5. Hypoxia induces IL-11 secretion of NSCLC cells by HIF-1. (A) A549 or H1299 cells were cultured under hypoxic $\left(0.5 \% \mathrm{O}_{2}\right)$ or normoxic conditions for 48 hours. Then, HIF-1 $\alpha$ expression was determined by western blot assay. (B and C) A549 cells were cultured under hypoxic conditions $\left(0.5 \% \mathrm{O}_{2}\right)$ or normoxic conditions for the indicated time. Then, IL-11 expression was determined by RT-qPCR (B) or ELISA assay (C). The data are shown as the means \pm SD. (D and E) H1299 cells were cultured under hypoxic conditions $\left(0.5 \% \mathrm{O}_{2}\right)$ or normoxic conditions for the indicated time. Then, IL-11 expression was determined by RT-qPCR (D) or ELISA assay (E). The data are shown as the means \pm SD. (F and G) A549 or H1299 cells were treated with either DFX $(100 \mu \mathrm{M})$ or DMOG $(1 \mathrm{mM})$ for 48 hours under normoxic conditions. Then, IL-11 expression was determined by RT-qPCR and ELISA. The data are shown as the means \pm SD. $*, \mathrm{P}<0.05, * *, \mathrm{P}<0.01$. 
A

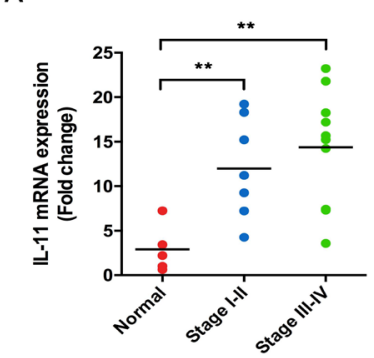

D

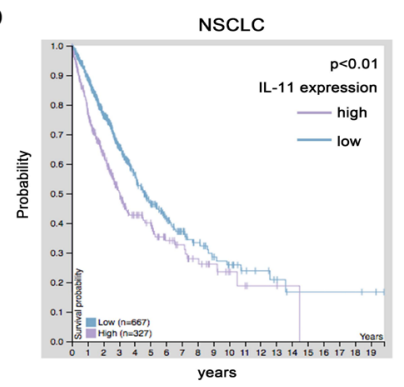

B

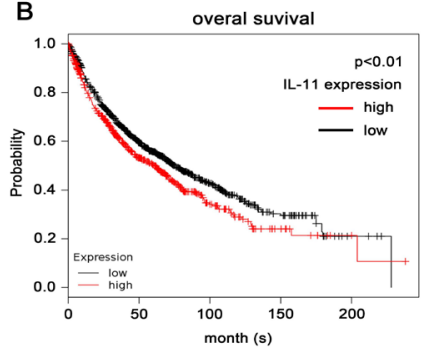

$\mathrm{E}$

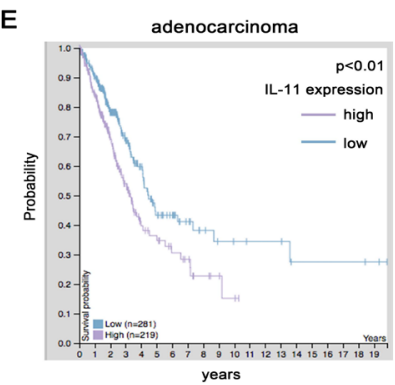

C

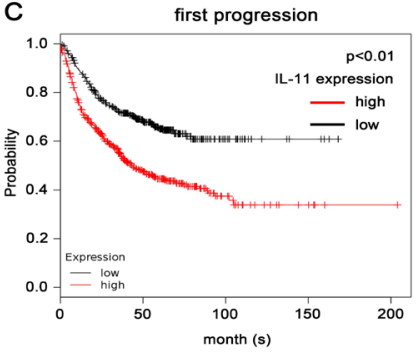

$\mathbf{F}$

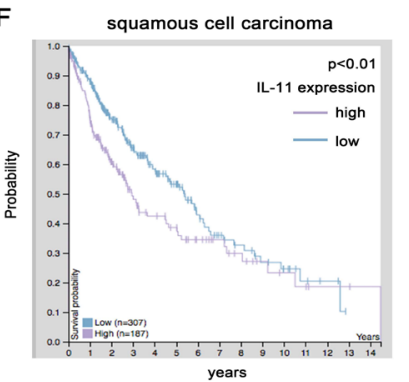

Fig. 6. IL-11 is upregulated in NSCLC and correlates with poor prognosis. (A) cDNA from 18 patients representing all disease stages and 5 normal lung samples were analyzed for IL-11 levels by qRT-PCR. The data are shown as the means $\pm \mathrm{SD} .{ }^{*}, * *, \mathrm{P}<0.01$. Kaplan-Meier overall survival analysis (B) and first progression (C) of IL-11 expression in NSCLC from a public data set (http://kmplot.com/analysis/index. php?p=service\&cancer=lung). Cutoff values were set to 76 (B), 50 (C). Kaplan-Meier overall survival analysis of IL-11 expression in NSCLC (D), adenocarcinoma (E) and squamous cell carcinoma (F) from Human Protein Atlas available (www.proteinatlas.org). Expression cutoffs were set to 0.9 FPKM (D), 0.4 FPKM (E),1.1 FPKM (F).

onstrated significant downregulation of snail and slug in IL-11 knockdown cells (Fig. 3B, 3C and 3D).

Furthermore, A549 and H1299 cells overexpressing IL-11 exhibited upregulated vimentin and N-cadherin and downregulated E-cadherin, claudin-1 and ZO-1 (Fig. 3E-3G). IL-11 neutralizing antibody reversed IL-11-induced EMT profiles (Fig. 3E-3G). In agreement with above results, incubation of A549 and $\mathrm{H} 1299$ cells with IL-11 also upregulated vimentin and N-cadherin and downregulated E-cadherin, claudin-1 and ZO-1 (Fig. 3E-3G). Taken together, these results indicate that IL-11 is an EMT promoter in NSCLC cells.

\section{IL-11 activates STAT3 and AKT pathway in NSCLC cells}

IL-11 binds to its specific transmembrane receptor, IL-11 receptor alpha (IL-11Ra), and the dimeric complex interacts with GP130 to activate downstream signaling [14]. We then turned to investigate the potential oncogenic pathway that IL11 activated in NSCLC. We first examined ERK, which participates in cell proliferation and invasion of tumor cells [15]. Results showed that suppression of IL-11 expression had no impact on ERK phosphorylation in A549 or H1299 cells (Fig. 4A). PI3K-AKT pathway plays a crucial role in EMT of cancer [16]. Given IL-11 promotes EMT in our experimental model, we examined IL-11 effects on AKT activation. As we expected, although total AKT expression remained unchanged, IL-11 inhibition suppressed phosphorylation of AKT on Ser473 and Thr308 in both A549 and H1299 cells (Fig. 4A). STAT family proteins are the major effectors of GP130 signaling [17]. We examined STAT3 and STAT5 activation status after IL-11 suppression. We observed that suppression of IL-11 did not alter the expression of pSTAT5 in A549 and H1299 cells, while pSTAT3 (Ser727) was significantly downregulated after IL-11 inhibition 
(Fig. 4A). Furthermore, sections from A549 tumors showed that shIL11 suppressed STAT3 phosphorylation in vivo (Fig. 4B). Taken together, these results indicate that IL-11 positively regulates AKT and STAT3 in NSCLC cells.

Hypoxia induces IL-11 secretion of NSCLC cells by HIF-1

Recent studies indicated the link between IL-11 and hypoxia [18]. However, the role of IL-11 in the hypoxic response of NSCLC is largely unknown. To explore the impacts of hypoxia on IL-11 expression, we first cultured A549 and H1299 cells under hypoxic conditions (0.5\% $\mathrm{O}_{2}$ ). Western blot analysis demonstrated that hypoxia stimulation induced HIF $1 \alpha$ expression in both A549 and H1299 cells (Fig. 5A). Furthermore, we observed that A549 cell upregulated IL-11 mRNA under hypoxic stress (Fig. 5B). Increased secretion of IL-11 was confirmed by ELISA assay (Fig. 5C). Consistent with the above results, similar data were obtained from H1299 cells. Hypoxia induced IL-11 mRNA expression and IL-11 secretion of H1299 cells (Fig. 5D and 5E).

Deferoxamine (DFX) and Dimethyloxalylglycine (DMOG) block HIF-1 degradation and promote HIF-1 accumulation. We incubated A549 and H1299 cells with DFX or DMOG for 24 hours, then examined IL-11 expression. The results showed that either DFX or DMOG treatment significantly increased IL-11 mRNA expression and secretion (Fig. 5F and 5G). Taken together, these results indicate that hypoxia induces IL-11 expression in NSCLC cells by HIF-1 and may play a crucial role in the hypoxia-induced response.

\section{IL-11 is upregulated in NSCLC and correlates with poor prognosis}

Previous data indicated the oncogenic role of IL-11 in NSCLC cells. To extend these observations into a clinicopathologically relevant setting, we analyzed IL-11 expression by RT-qPCR in cDNA from 18 NSCLC patients and 5 normal lung samples. The results showed that IL-11 expression was significantly upregulated in tumor samples than normal lung samples (Fig. 6A). Furthermore, public data from an online database $[19,20]$ revealed that high levels of IL-11 expression correlated with worse prognosis (Fig. 6B-6F).

\section{Discussion}

Non-small cell lung cancer is one of the leading causes of deaths from cancer worldwide. Therefore, fully understanding the mechanisms of NSCLC progression leads to improvements in diagnostics and treatments [21]. IL-11 has gained less attention compared with its relatives, the IL-6 family, especially in NSCLC. In the current study, we explored the role of IL-11 systematically to provide a deep understanding of IL-11 in NSCLC. Our results provide evidence that IL-11 is one of the crucial hypoxia players and promotes tumor progression in NSCLC.

Upregulation of IL-11 expression was observed in NSCLC samples compared with normal tissue samples, which indicates that high levels of IL-11 may relate to the progression of NSCLC. Public data from online indicate that high expression of IL-11 correlates with poor prognosis. Furthermore, inhibition of IL-11 in NSCLC cell lines (A549 and H1299) suppressed cell proliferation, invasion and migration in vitro. Furthermore, IL-11 depletion significantly impaired tumorigenesis in vivo and prolonged mice survival. Collectively, these results indicate an oncogenic role in NSCLC. It was recently reported that IL-11 is a potential oncogene in gastrointestinal and hepatocellular cancers [22]. Our report is consistent with these results and imply that the regulatory effect of IL-11 on tumor progression may exist for a broad spectrum of malignant diseases.

IL-11 primarily exerts its effects by interacting with IL-11R $\alpha$ and GP130 [14]. However, the downstream effector may be variant in different tissue contexts [23]. Our study showed that although IL-11 has no impact on phosphorylation of ERK1/2 and STAT5, pSTAT3 and AKT were severely upregulated. Furthermore, IL-11 positively regulates phosphorylation of 
STAT3 on Ser727, but not Tyr705. These results confirm the complexity function of IL-11 in different tumors.

Hypoxia is a universal driver of cancer that influences almost every aspect of the biology of tumors. One approach to targeting hypoxia is to inhibit molecules involved in the survival of hypoxia cells [24]. The results presented in this paper uncovered IL-11 as a crucial hypoxia player in NSCLC. IL-11 is overexpressed under hypoxia in NSCLC cells. Inhibition of HIF-1 $\alpha$ by either DFX or DMOG could also upregulate IL-11 secretion, which indicates IL-11 is induced by HIF1 $\alpha$ activation. A recent study in prostate cancer indicates that hypoxic cancer cells mediate the autocrine production of IL-11 [18]. Together with this study, our results indicate IL-11 as a potential target of HIF-1 and participate in the hypoxia response of NSCLC.

EMT enables tumor cells to gain invasion and migration capabilities, and this phenomenon has been reported in a variety of malignancies, such as skin, head and neck, breast, ovarian, liver, colon and prostate cancer. In NSCLC, EMT contributes not only to invasion and distal metastasis but also chemoresistance [25-27]. However, the regulation of EMT in NSCLC is far from fully understood. In this study, we found that IL-11 positively regulates EMT in NSCLC cells. Suppression of IL-11 induced expression of epithelial makers, such as E-cadherin, claudin-1 and ZO-1, and downregulated mesenchymal markers, such as vimentin and $\mathrm{N}$-cadherin. Consistent with these results, overexpression of IL-11 reversed the effects.

\section{Conclusion}

In summary, the results of our study indicate that IL-11 is a potential oncogene upregulated in NSCLC and correlates with poor prognosis and may participate in the hypoxia response and EMT. We propose IL-11 as a potential therapeutic target and prognostic marker for NSCLC. However, further investigation is required.

\section{Acknowledgements}

This work was supported by the National Natural Science Foundation of China, grants No. 81402174 and No. 81602026.

\section{Disclosure Statement}

No conflict of interest exists.

\section{References}

1 Dranoff G: Cytokines in cancer pathogenesis and cancer therapy. Nature reviews Cancer 2004;4:11-22.

-2 Siegel RL, Miller KD, Jemal A: Cancer Statistics, 2017. CA Cancer J Clin 2017;67:7-30.

-3 Azad N, Rojanasakul Y, Vallyathan V: Inflammation and lung cancer: roles of reactive oxygen/nitrogen species. J Toxicol Environ Health B Crit Rev 2008;11:1-15.

4 Lippitz BE: Cytokine patterns in patients with cancer: a systematic review. Lancet Oncol 2013;14:e218-e228.

5 Putoczki TL, Thiem S, Loving A, Busuttil RA, Wilson NJ, Ziegler PK, Nguyen PM, Preaudet A, Farid R, Edwards KM, Boglev Y, Luwor RB, Jarnicki A, Horst D, Boussioutas A, Heath JK, Sieber OM, Pleines I, Kile BT, Nash A, Greten FR, McKenzie BS, Ernst M: Interleukin-11 is the dominant IL- 6 family cytokine during gastrointestinal tumorigenesis and can be targeted therapeutically. Cancer Cell 2013;24:257-271.

6 Chen C, Zhao M, Tian A, Zhang X, Yao Z, Ma X: Aberrant activation of Wnt/beta-catenin signaling drives proliferation of bone sarcoma cells. Oncotarget 2015;6:17570-17583. 


\section{Cellular Physiology Cell Physiol Biochem 2018;45:2213-2224

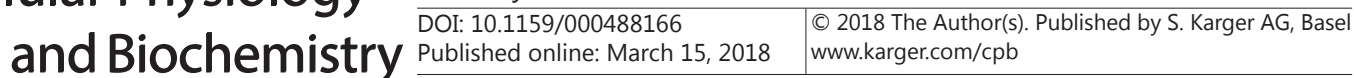

7 Huang Y, Zhao M, Xu H, Wang K, Fu Z, Jiang Y, Yao Z: RASAL2 down-regulation in ovarian cancer promotes epithelial-mesenchymal transition and metastasis. Oncotarget 2014;5:6734-6745.

8 Hanahan D, Weinberg RA: The hallmarks of cancer. Cell 2000;100:57-70.

-9 Hanahan D, Weinberg RA: Hallmarks of cancer: the next generation. Cell 2011;144:646-674.

10 Lamouille S, Xu J, Derynck R: Molecular mechanisms of epithelial-mesenchymal transition. Nat Rev Mol Cell Biol 2014;15:178-196.

11 Thiery JP: Epithelial-mesenchymal transitions in tumour progression. Nature Rev Cancer 2002;2:442-454.

12 Kalluri R, Weinberg RA: The basics of epithelial-mesenchymal transition. J Clin Invest2009;119:1420-1428.

-13 Thiery JP, Acloque H, Huang RY, Nieto MA: Epithelial-mesenchymal transitions in development and disease. Cell 2009;139:871-890.

14 Johnstone CN, Chand A, Putoczki TL, Ernst M: Emerging roles for IL-11 signaling in cancer development and progression: Focus on breast cancer. Cytokine Growth Factor Rev 2015;26:489-498.

15 Samatar AA, Poulikakos PI: Targeting RAS-ERK signalling in cancer: promises and challenges. Nature reviews Drug Discov 2014;13:928-942.

16 Larue L, Bellacosa A: Epithelial-mesenchymal transition in development and cancer: role of phosphatidylinositol 3[prime] kinase//AKT pathways. Oncogene 0000;24:7443-7454.

17 Silver JS, Hunter CA: gp130 at the nexus of inflammation, autoimmunity, and cancer. J Leuk Biol 2010;88:1145-1156.

18 Onnis B, Fer N, Rapisarda A, Perez VS, Melillo G: Autocrine production of IL-11 mediates tumorigenicity in hypoxic cancer cells. J Clin Invest 2013;123:1615-1629.

19 Gyorffy B, Surowiak P, Budczies J, Lanczky A: Online survival analysis software to assess the prognostic value of biomarkers using transcriptomic data in non-small-cell lung cancer. PloS one 2013;8:e82241.

20 Uhlen M, Fagerberg L, Hallstrom BM, Lindskog C, Oksvold P, Mardinoglu A, Sivertsson A, Kampf C, Sjostedt E, Asplund A, Olsson I, Edlund K, Lundberg E, Navani S, Szigyarto CA, Odeberg J, Djureinovic D, Takanen JO, Hober S, Alm T, Edqvist PH, Berling H, Tegel H, Mulder J, Rockberg J, Nilsson P, Schwenk JM, Hamsten M, von Feilitzen K, Forsberg M, Persson L, Johansson F, Zwahlen M, von Heijne G, Nielsen J, Ponten F: Proteomics. Tissue-based map of the human proteome. Science (New York, NY) 2015;347:1260419.

21 Reck M, Heigener DF, Mok T, Soria J-C, Rabe KF: Management of non-small-cell lung cancer: recent developments. Lancet 2013;382:709-719.

22 Yuan JH, Yang F, Wang F, Ma JZ, Guo YJ, Tao QF, Liu F, Pan W, Wang TT, Zhou CC, Wang SB, Wang YZ, Yang Y, Yang N, Zhou WP, Yang GS, Sun SH: A long noncoding RNA activated by TGF-beta promotes the invasionmetastasis cascade in hepatocellular carcinoma. Cancer Cell 2014;25:666-681.

23 Xu DH, Zhu Z, Wakefield MR, Xiao H, Bai Q Fang Y: The role of IL-11 in immunity and cancer. Cancer Lett 2016;373:156-163.

24 Wilson WR, Hay MP: Targeting hypoxia in cancer therapy. Nature reviews Cancer 2011;11:393-410.

25 Xiao D, He J: Epithelial mesenchymal transition and lung cancer. J Thor Dis 2010;2:154-159.

-26 Fischer KR, Durrans A, Lee S, Sheng J, Li F, Wong STC, Choi H, El Rayes T, Ryu S, Troeger J, Schwabe RF, Vahdat LT, Altorki NK, Mittal V, Gao D: Epithelial-to-mesenchymal transition is not required for lung metastasis but contributes to chemoresistance. Nature 2015;527:472-476.

-27 Wang D, Shi W, Tang Y, Liu Y, He K, Hu Y, Li J, Yang Y, Song J: Prefoldin 1 promotes EMT and lung cancer progression by suppressing cyclin A expression. Oncogene 2017;36:885-898. 